\title{
Indices Using External Measurements for Assessing Fat Deposition of Adult Feral Raccoons (Procyon lotor) in Kanagawa Prefecture, Japan
}

\author{
Takuya KATO ${ }^{1) *}$, Taiki $\mathrm{UNO}^{1)}$, Yoshiyuki FUJIOKA ${ }^{1)}$ and Shin-ichi HAYAMA ${ }^{1)}$ \\ ${ }^{1)}$ Laboratory of Wildlife Medicine, Nippon Veterinary and Life Science University, 1-7-1, Kyonan-cho, Musashino, Tokyo 180-8602, \\ Japan
}

(Received 27 April 2011/Accepted 26 September 2011/Published online in J-STAGE 7 October 2011)

\begin{abstract}
We examined the use of external measurements and relative fat deposition of adult feral raccoons (Procyon lotor) to develop relative indices of body fat deposition in post-growth feral raccoons. From March 2006 to March 2010, 288 adult raccoon carcasses (110 males, 178 females) collected in Kanagawa Prefecture, Japan, which were determined to be 24 months old, were subjected to external measurements of body weight (BW), girth measurement (GM), and body mass index (BMI). To assess relative body fat deposition, we visually classified abdominal subcutaneous fat into three grades (Visible Fat Index [VFI]: I-III). Significant differences in the means of BW (both sexes: $P<0.01$ ), GM (females: $P<0.05$, males: $P<0.01$ ), and BMI (both sexes: $P<0.01$ ) were detected between seasons. Notably, the means of BW, GM, and BMI (all, both sexes: $P<0.01$ ) differed significantly between VFI grades. However, by discriminant analysis with $\mathrm{BW}, \mathrm{GM}$, and $\mathrm{BMI}$ as independent variables, we obtained a significant discriminant function (both sexes: $P<0.01$ ) for distinguishing VFI I from higher VFI grades, but no significant equation was obtained for distinguishing between VFI II and VFI III. Based on the obtained structure matrix of discriminant analysis, BMI was the most valuable component for the discrimination of VFI grades. Thus, we conclude that BMI is a suitable complementary index for assessing relative body fat deposition of adult feral raccoons in Kanagawa Prefecture and may be generalizable to populations in other areas.

KEY WORDS: body mass index, external measurement, fat deposition, Procyon lotor, raccoon.
\end{abstract}

doi: 10.1292/jvms.11-0205; J. Vet. Med. Sci. 74(2): 181-188, 2012

Assessment of the nutritional status of wild animals is one of the basic components for understanding seasonal forage [39, 40], survival strategies [34, 39, 40], and reproductive success, including age at primiparity [30], breeding interval [8], pregnancy rate $[7,8]$, litter size $[7,8]$, and cub survival $[3,7]$. The nutritional status of wild animals fluctuates seasonally in response to environmental factors such as food availability $[39,40]$. In areas with severe winter climate, wild animals begin to accumulate body fat reserves in the autumn to overcome low temperatures and food shortage $[34,39,40]$. Additionally, when wild animals have access to abundant nutrition, reproductive success and population survival are correspondingly high $[3,7,8,30]$. Previous studies have indicated that body fat deposition represents a good indicator of the nutritional status of wild mammals [14]. As nutritional status may have affects on wildlife population dynamics, examining the body fat deposition of animals may aid in the adaptive management of target wildlife populations.

To assess body fat deposition in wild animals, a number of direct and indirect methods have been reported. The most accurate index of body fat deposition is considered to be the amount of stored lipids, which can be quantified by direct chemical analysis of homogenates prepared from whole carcasses $[4,9,10,18,27,34,40]$. Despite having high accu-

\footnotetext{
* Correspondence to: Kato, T., Laboratory of Wildlife Medicine, Nippon Veterinary and Life Science University, 1-7-1, Kyonancho, Musashino, Tokyo 180-8602, Japan.

e-mail: takuya-v-magna@mub.biglobe.ne.jp

(C)2012 The Japanese Society of Veterinary Science
}

racy, this method is expensive and time consuming, and it is not suitable for the rapid examination of a large number of samples. As an alternative to direct analysis, several indirect methods for estimating body fat deposition have also been developed. Total body fat has been predicted from measurements of body fat from specific areas of the body, such as subcutaneous and perivisceral fat, as Cavallini [6] did in the red fox (Vulpes vulpes).

In recent years, bioelectrical impedance analysis (BIA) has been introduced for estimating body fat deposition in the American black bear (Ursus americanus) [9, 17, 30], Brown bear (U. arctos) [9, 17], Polar bear (U. maritimus) [3, 9], and red fox ( $V$. vulpes) [27]. In addition, several studies have evaluated body fat deposition using non-lethal methods based on the relationship between external and fat deposit measurements in a number of wild mammal species, including the American black bear [5, 30], red fox [27], coyote (Canis latrans) [18], and Tsushima leopard cat (Prionailurus bengalensis euptiluurus) [15].

Several studies have also examined body fat deposition in raccoons (Procyon lotor). For example, using a method involving the measurement of body weight, Mech et al. [28] reported that raccoons at the edge of their distribution range can lose up to $50 \%$ of their body weight from autumn to spring. However, body weight was also considered to increase as a result of juvenile growth, as raccoons do not reach full growth until their second year, as shown in Minnesota [28], Alabama [21], South Texas [12], and Hokkaido [1]. Although external measurements such as body weight (BW), girth measurement (GM), and body mass index 
(BMI) have been widely used as convenient indices to evaluate the nutritional status of raccoons $[11,21,26,28,29$, $33]$, BIA has been demonstrated to be a more accurate method than morphological indices to assess the body fat of raccoons [31]. Other specialized methods, including ultrasonic measurements [37] and serum leptin assays [36] have also been used for estimating the body condition of raccoons.

The raccoon is native to North America and is considered an invasive alien species in Japan. Recently, the raccoon has naturalized and expanded its distribution to include numerous prefectures in Japan, which is of concern as feral raccoons are considered to have negative impacts on native ecosystems, agriculture, and public health [19]. As these issues may become more serious with the growing populations of naturalized raccoons, scientific and practical management approaches are necessary to eradicate the feral raccoon population in Japan. By examining captured raccoons in Hokkaido [2] and Kanagawa Prefecture [24] in Japan, it was determined that the breeding season, age-specific pregnancy rate, and litter size of feral raccoons are similar to those of North American populations. However, in both Kanagawa Prefecture and Hokkaido populations, the effect of intrinsic factors, such as body fat deposition, on fluctuations in the reproductive characteristics of feral raccoons was not demonstrated. The raccoon is known to be an adaptive mammal and have a wide food habitat in North America [38]. Nevertheless, the nutritional status of raccoons is reported to correlate with environmental factors, including food availability [21], winter severity [32], and population density [33], and has an effect on both reproduction $[11,21]$ and mortality $[28,32,33]$. Thus, estimation of the body fat deposition of feral raccoons has become a key criterion for discussion when formulating an adaptive eradication program for feral raccoons as it might be an indicator for reproductive status and/or mortality.

The BIA method is accurate, simple, and rapid, but is expensive and the results can be influenced by weather and pre-existing condition of the animal [30]. Furthermore, portable BIA equipment, which was used in several previous studies $[3,9,17,27,30,31]$, is not readily available in Japan, so BIA methods for measuring body fat deposition are not currently suitable for wildlife management here. In fact, although over 1,000 raccoons per year have been captured in efforts to reduce the feral raccoon population size in Kanagawa Prefecture [23], BIA or other specialized methods have not been used for examination of body fat deposition for any of the captured raccoons. Although BIA may become a more common method in the future, alternative methods to assess the relative fat deposition of captured raccoons are urgently needed at present. External measurements represent one promising approach as they may be able to assess relative body fat deposition of feral raccoons easily and inexpensively. However, no studies have examined the relationship between body fat deposition and external measurements for feral raccoons in Japan.

The purpose of this study therefore was to develop a com- plementary method for estimating body fat deposition in post-growth feral raccoons in Kanagawa Prefecture. We first examined whether adult feral raccoons ( 24 months old) exhibited seasonal fluctuations in external measurements of BW, GM, and BMI. We then examined the relationship between these external measurements and amount of body fat to develop a method for assessing body fat deposition from external measurements.

\section{MATERIALS AND METHODS}

Kanagawa Prefecture $\left(\mathrm{N} 35^{\circ} \mathrm{E} 139^{\circ}\right)$ is located on the southwest edge of the Kanto Plain, Japan (Fig. 1). In this region, the climate is temperate with dry winters and humid summers, and the mean annual temperature and sunshine duration are $15.5^{\circ} \mathrm{C}$ and $1920.6 \mathrm{hrs}$, respectively. For our study, all of the raccoon specimens were collected during pest control activities or by the Kanagawa Raccoon Control Program under the initiative of the Invasive Alien Species Act. All raccoons were captured using box traps (Havahart Live Animal Cage Trap Model 1089; Woodstream, Lititz, PA, U.S.A.) and were euthanized by pentobarbital sodium injection or $\mathrm{CO}_{2}$ inhalation, according to the Guidelines for the Management of Invasive Alien Species [20].

We collected raccoon carcasses in Kamakura, Yokosuka, Yokohama, and Hayama in Kanagawa Prefecture from March 2006 to March 2010 (Fig. 1). To analyze seasonal body fat deposition of raccoons, external measurement data were compared among the four seasons, which were designated as follows: spring (March to May), summer (June to August), autumn (September to November), and winter (December to February). The respective values for average seasonal temperature between 2006 and 2010 were 14.1, $24.0,18.5$, and $7.6^{\circ} \mathrm{C}$. To identify post-growth raccoons, we estimated their ages by cranial sutures and selected raccoons $>24$ months old, which were considered adult $[13,22]$. A total of 110 adult males (30 individuals in spring, 30 in summer, 20 in autumn, and 30 in winter) and 178 adult females

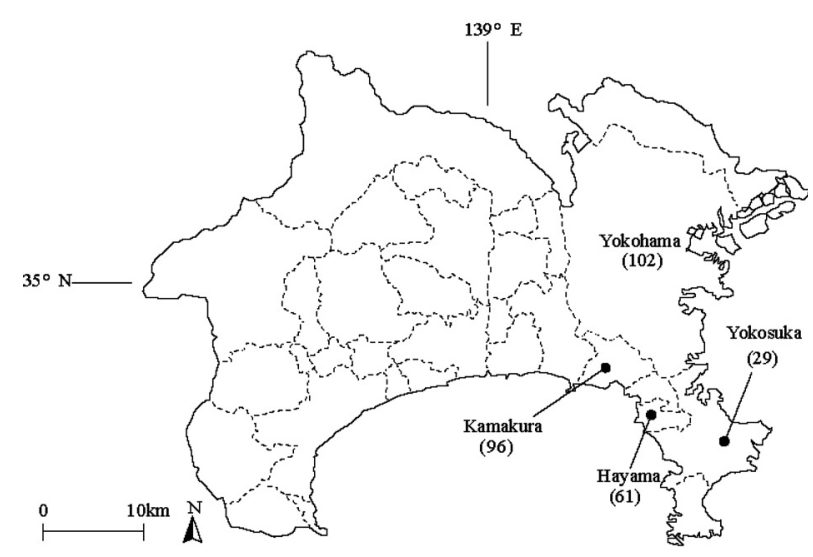

Fig. 1. Location of the study area for feral raccoons in Kanagawa Prefecture, Japan, 2006-2010. Numbers in parentheses represent the total number of raccoons collected from the area. 
(45 individuals in spring, 46 in summer, 56 in autumn, and 31 in winter) were used for analysis, and we confirmed that 25 females were pregnant (18 individuals in spring, 2 in summer, and 5 in winter) by observing fetuses in utero. We used external measurement data of BW $(\mathrm{g})$, body length (BL, mm), and GM (mm). The BW of pregnant females was calculated by excluding fetus weight. GM was taken at the posterior end of the sternum immediately anterior to the xiphoid process [26]. BMI was calculated as described in previous studies $[31,36]$, using the following equation:

$$
\mathrm{BMI}=\mathrm{BW}(\mathrm{kg}) / \mathrm{BL}(\mathrm{m})^{2}
$$

Visible fat index (VFI): Due to difficulties in the direct measurement of body fat deposition because of a lack of suitable equipment, we assessed relative body fat deposition using visual grades of body fat. In a previous study by Johnson [21], subcutaneous fat of the abdomen of raccoons was assessed visually and classified from grades 1 to 3 . Following this classification system, we assessed abdominal subcutaneous fat using a visible fat index (VFI) consisting of three grades (I-III). To determine the VFI, a median incision was made in the abdomen of all 288 specimens, and the incision site was then opened and photographed with a digital camera.

Based on these photographs, fat deposition was assessed visually and independently of external measurements according to the criteria for the three grades (Fig. 2). To limit inter-observer variation and seasonal bias, all specimens were measured by the same individual and were randomly sorted. Subcutaneous fat deposition was visually assessed according to the following VFI grades.

Grade I: No or few fat deposits are visible in the abdominal subcutaneous region (A).

Grade II: Fat deposits are partly visible in the abdominal subcutaneous region (B).

Grade III: A large amount of fat deposits are visible throughout the abdominal subcutaneous region (C).

The assessment of VFI grades for all specimens was independently repeated three times. As no specimens were classified into all of the three VFI grades, the VFI grade for which at least two results coincided was reported in this study.

Statistical analysis: All statistical analyses were performed using SPSS 16.0 J for Windows (SPSS, Chicago, IL, U.S.A.). We considered $P$ values $<0.05$ to be statistically significant. The differences among the mean values of BW, GM, and BMI in each season or VFI grade were analyzed using Tukey's post-hoc test. To examine whether raccoons could be classified into VFI grades I, II, and III from external measurements, discriminant analysis was carried out using BW, GM, and BMI as independent variables according to previous studies $[15,16]$. The results of this analysis provided coefficients of each measurement for linear discriminant function 1, which distinguished VFI I from VFI II and VFI III fat deposit groups, and linear discriminant function 2, which classified raccoons into VFI II and VFI III fat

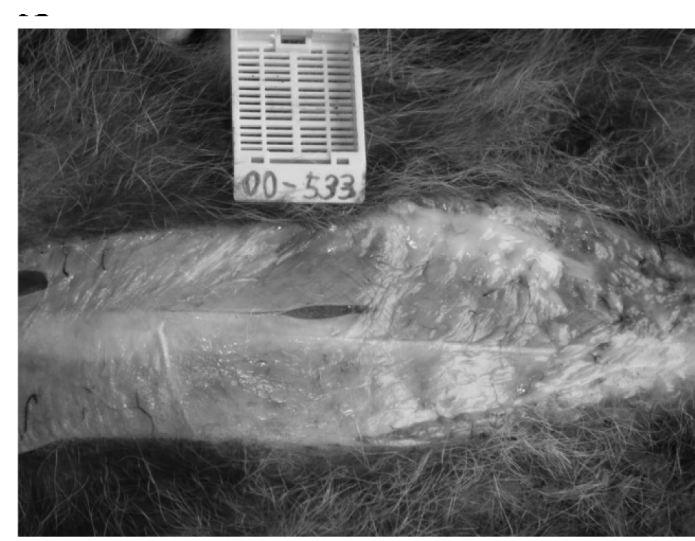

B

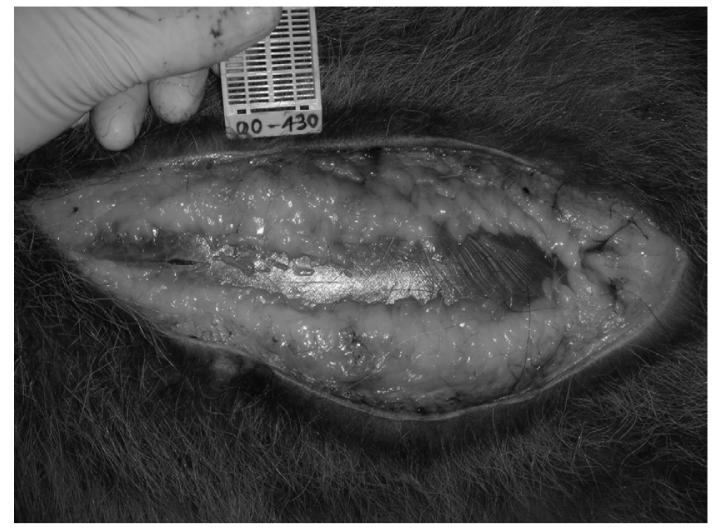

C

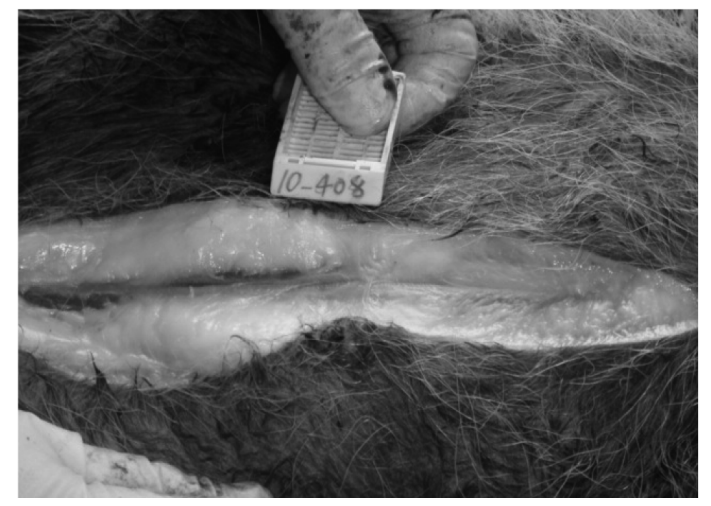

Fig. 2. Visible fat index (VFI) standards for feral raccoons in Kanagawa Prefecture. Subcutaneous fat deposition in the abdomen was visually assessed according to the following VFI grades: Grade I: No or few fat deposits are visible in the abdominal subcutaneous region (A) Grade II: Fat deposits are partly visible in the abdominal subcutaneous region (B). Grade III: A large amount of fat deposits are visible throughout the abdominal subcutaneous region $(\mathrm{C})$.

deposit groups. Structure matrix values processed by discriminant analysis were tested for the most useful component of external measurements. 


\section{RESULTS}

Seasonal variations in external measurements: The mean and standard deviation of the external measurements (BW, GM, and BMI) for the 288 feral raccoons collected in Kanagawa Prefecture based on sex and season are shown in Table 1. From the analysis of variance results, both sexes of adult raccoons displayed seasonal variations in the means of $\mathrm{BW}$ (females: $F=5.54, d f=3, \mathrm{p}<0.01$; males: $F=4.62, d f=3$, $P<0.01$ ), GM (females: $F=3.67, d f=3, P<0.05$; males: $F=4.73, d f=3, P<0.01$ ), and BMI (females: $F=11.25, d f=3$, $P<0.01$; males: $F=5.28, d f=3, P<0.01)$.

In females, the mean values of all external measurements in summer were significantly lower than those in autumn $(P<0.05)$. The means of BW and BMI in summer were also significantly lower than those in winter $(P<0.05)$, but the means of GM between summer and winter did not differ significantly $(P=0.06)$. Furthermore, only differences in the BMI were observed between spring and winter, with mean values being lower in spring than in winter $(P<0.05)$. In contrast, the means of all external measurements varied between spring and winter in males $(P<0.05)$. Additionally, significant differences in the means of GM and BMI were observed between spring and autumn $(P<0.05)$, and between summer and winter $(P<0.05)$, respectively.

Differences of external measurements by VFI grade: Figure 3 shows the differences in the mean values of the external measurements (BW, GM, and BMI) for each VFI grade for all 288 feral raccoons. The mean values of $\mathrm{BW}$ (females: $F=57.32, d f=2, P<0.01$; males: $F=45.21, d f=2$, $P<0.01$ ), GM (females: $F=25.81, d f=2, P<0.01$; males: $F=26.70, d f=2, P<0.01$ ), and BMI (females: $F=102.23$, $d f=2, P<0.01$; males: $F=71.20, d f=2, P<0.01)$ significantly differed between the three VFI grades in both sexes, with the means of all external measurements tending to increase at higher VFI grades. Significant differences in the means of all external measurements were observed between VFI I and VFI II (females: $P<0.01$, males: $P<0.05$ ), VFI II and VFI III (both sexes: $P<0.01$ ), and between VFI I and VFI III (both sexes: $P<0.01$ ).
Discriminant analysis for classifying raccoons into fat deposit groups: To classify raccoons into VFI I, VFI II, and VFI III groups, discriminant analysis was performed using BW, GM, and BMI as independent variables for each sex and age class (Table 2). We obtained the following discriminant functions, where $\mathrm{Z}$ is the discriminant score:

Adult female discriminant function 1:

$\mathrm{Z}=0.001(\mathrm{BW})-0.007(\mathrm{GM})+0.492(\mathrm{BMI})-8.098$

Adult female discriminant function 2:

$\mathrm{Z}=-0.001(\mathrm{BW})+0.033(\mathrm{GM})+0.093(\mathrm{BMI})-7.040$

Adult male discriminant function 1:

$\mathrm{Z}=0.001(\mathrm{BW})+0.001(\mathrm{GM})+0.431(\mathrm{BMI})-11.225$

Adult male discriminant function 2:

$\mathrm{Z}=0.001(\mathrm{BW})+0.033(\mathrm{GM})-0.322(\mathrm{BMI})-5.435$

Discriminant function 1 was obtained as a significant discriminant equation for males as well as females, and could successfully distinguish VFI I from VFI II and VFI III fat deposit groups $(P<0.01)$. However, discriminant function 2 was unable to classify raccoons into VFI II and VFI III fat deposit groups (females: $P>0.5$, males: $P>0.1$ ).

The total classification accuracies for each VFI group, as calculated using discriminant functions for data for each sex, were $70.2 \%$ in females and $77.3 \%$ in males (Table 2). In each sex group, the accuracy of VFI I classification was high (females: $84.2 \%$, males: $81.2 \%$ ), whereas that of VFI II (females: $50.9 \%$, males $72.1 \%$ ) and VFI III (females: $74.2 \%$, males $77.3 \%$ ) was slightly lower. Based on the structure matrix values obtained using the significant discriminant function, BMI was the most useful component of all external measurements for the discrimination of VFI grade in both sexes (Table 2).

\section{DISCUSSION}

Several studies have reported that BW of North American raccoons shows seasonal variation $[12,21,28,29,35,38]$. Northern raccoon populations in North America lose approximately $50 \%$ of their body weight during winter [28, $38]$, whereas raccoon populations at southern latitudes lose only $20-30 \% \mathrm{BW}[12,21]$. The winter weight losses of raccoon populations in Hokkaido, which is one area in Japan

Table 1. Seasonal changes in the means \pm SD of body weight (BW), girth measurement (GM), and body mass index $(\mathrm{BMI})$ of adult feral raccoons $(\mathrm{n}=288)$ in Kanagawa Prefecture

\begin{tabular}{llllll}
\hline Sex & Season & N & \multicolumn{1}{c}{ BW $(\mathrm{g})$} & \multicolumn{1}{c}{ GM $(\mathrm{mm})$} & BMI $\left(\mathrm{kg} / \mathrm{m}^{2}\right)$ \\
\hline Female $^{\mathrm{a})}$ & Spring & 45 & $5457.82 \pm 981.73$ & $387.42 \pm 44.62$ & $\left.18.62 \pm 2.82^{\mathrm{b}}\right)$ \\
& Summer & 46 & $5196.45 \pm 769.10^{\mathrm{b}, \mathrm{c}}$ & $372.11 \pm 39.98^{\mathrm{b})}$ & $17.31 \pm 2.18^{\mathrm{c}, \mathrm{d})}$ \\
& Autumn & 56 & $5899.91 \pm 1121.54^{\mathrm{b})}$ & $398.43 \pm 44.18^{\mathrm{b})}$ & $19.51 \pm 2.88^{\mathrm{c})}$ \\
& Winter & 31 & $5922.16 \pm 1036.69^{\mathrm{c})}$ & $397.23 \pm 39.44$ & $20.77 \pm 2.77^{\mathrm{b}, \mathrm{d})}$ \\
\hline \multirow{2}{*}{ Male } & Spring & 30 & $6149.83 \pm 907.52^{\mathrm{b})}$ & $371.90 \pm 30.89^{\mathrm{b}, \mathrm{c})}$ & $19.57 \pm 2.13^{\mathrm{b})}$ \\
& Summer & 30 & $6657.50 \pm 1270.11$ & $395.13 \pm 44.71$ & $19.32 \pm 2.36^{\mathrm{c}}$ \\
& Autumn & 20 & $7052.75 \pm 1200.33$ & $412.75 \pm 32.36^{\mathrm{b})}$ & $20.88 \pm 2.20$ \\
& Winter & 30 & $7349.17 \pm 1614.83^{\mathrm{b})}$ & $401.57 \pm 46.71^{\mathrm{c}}$ & $21.59 \pm 3.03^{\mathrm{b}, \mathrm{c})}$ \\
\hline
\end{tabular}

a) BW of pregnant females was calculated by excluding fetus weight. BMI was also calculated following this parameter. The same symbols (b, c, and d) appear in the measurement for each sex group for which a significant differences $(P<0.05)$ was observed by Tukey's post-hoc test. 

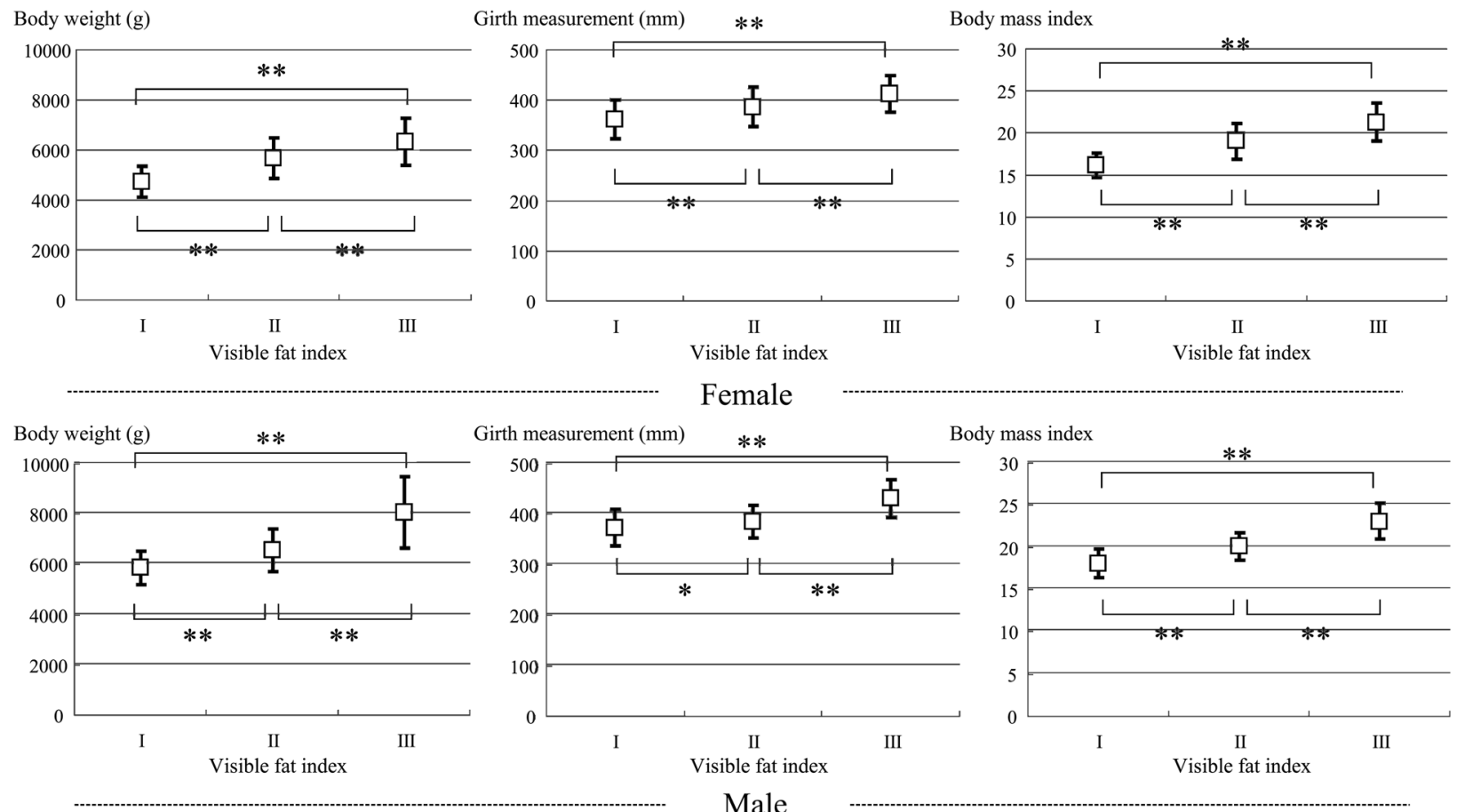

Male

Fig. 3. Means of external measurements versus seasonal Visible Fat Index (VFI) grades of female (above) and male (below) feral adult raccoons in Kanagawa Prefecture. Both body weight and body mass index of pregnant females were calculated by excluding the body weight of fetuses. *: Significant difference of $P<0.05$ between different VFI grades (Tukey's post-hoc test). **: Significant difference of $P<0.01$ between different VFI grades (Tukey's post-hoc test).

Table 2. Discriminant functions, Wilks $\lambda, P$ values, structure matrixes values, and accuracy of discriminant analysis for classifying adult raccoons into three Visible Fat Index (VFI) grades using body weight (BW), girth measurement (GM), and body mass index (BMI)

\begin{tabular}{|c|c|c|c|c|c|}
\hline & & \multicolumn{2}{|c|}{ Female $^{a)}$} & \multicolumn{2}{|c|}{ Male } \\
\hline & & Function $1^{\text {b) }}$ & Function $2^{\text {b) }}$ & Function $1^{\text {b) }}$ & Function $2^{\text {b) }}$ \\
\hline \multicolumn{2}{|l|}{$\mathrm{N}$} & \multicolumn{2}{|r|}{178} & \multicolumn{2}{|c|}{110} \\
\hline Wilks $\lambda$ & & 0.45 & 0.99 & 0.42 & 0.97 \\
\hline$P$ value & & $<0.01$ & $0.5<$ & $<0.01$ & $0.1<$ \\
\hline \multirow[t]{4}{*}{ Accuracy (\%) } & VFI I & \multicolumn{2}{|r|}{84.2} & \multicolumn{2}{|c|}{81.2} \\
\hline & VFI II & \multicolumn{2}{|r|}{50.9} & \multicolumn{2}{|c|}{72.1} \\
\hline & VFI III & \multicolumn{2}{|r|}{74.2} & \multicolumn{2}{|c|}{80.0} \\
\hline & Total & \multicolumn{2}{|r|}{70.2} & \multicolumn{2}{|c|}{77.3} \\
\hline \multirow[t]{3}{*}{ Structure matrix values $\left.{ }^{c}\right)$} & BW & \multicolumn{2}{|r|}{0.731} & \multicolumn{2}{|c|}{0.747} \\
\hline & GM & \multicolumn{2}{|r|}{0.488} & \multicolumn{2}{|c|}{0.590} \\
\hline & BMI & \multicolumn{2}{|r|}{0.977} & \multicolumn{2}{|c|}{0.960} \\
\hline
\end{tabular}

a) BW of pregnant females was calculated by excluding the fetus weight. BMI was also calculated following this parameter. b) VFI I was distinguished from VFI II or VFI III using Function 1, and VFI II was distinguisherd from VFI III using discriminant Function 2. c) The values show the correlation with the discriminant function, where the highest correlation is shown by the items with the highest values. Only the values obtained using Function 1 (significant) are shown for each sex.

where raccoons have been introduced, were reported to be $28 \%$ and $25 \%$ in adult females and males, respectively [1]. Weight fluctuations of mammals are characterized by BW increases during autumn due to hyperphasia and by decreases during winter due to winter severity $[25,28,32$, $34,39,40]$. In the present study, we were unable to examine the winter losses of BW in raccoons directly. Nevertheless, the seasonal fluctuation patterns of BW in the Kanagawa 
females collected in this study seemed to differ from those of Hokkaido, where raccoons of both sexes have the lowest BW during spring and gradually gain weight beginning in summer [1]. Although the male raccoons from Kanagawa showed a similar fluctuation pattern of BW as raccoons in Hokkaido, the Kanagawa females had the lowest BW during summer. Prestrud and Nilssen [34] suggested that the poor nutritional condition of post-reproductive females of Arctic foxes (Alopex lagopus) resulted from the increased energy demands of extended parental care of pups. Although our study did not distinguish between female reproductive stages, parturition of raccoons in Kanagawa occurs over longer periods than that of Hokkaido populations, with the proportion of parous females reaching approximately $80 \%$ in feral raccoons in Kanagawa [24]. Therefore, we consider that in addition to seasonal variation, BW fluctuation of feral raccoons is also related to female breeding stage.

In a previous study of GM in North American raccoons, no significance difference in the mass to girth ratio was identified between seasons [26]. In the present study, we demonstrated that not only mass, but also girth, varies by season. It is possible that the previous failure to detect seasonal fluctuation in the mass to girth ratio was due to similar changes in both mass and girth during seasonal fat deposition. The body regions of mammalian fat deposition and the order in which they accumulate fat vary between animal species [14]. Pitt et al. [31] reported that the distribution of body fat deposition in raccoons was around $96 \%$ subcutaneous fat. Here, the girth of adult feral raccoons, which is influenced by abdominal subcutaneous fat deposits, was found to vary by season, although little is known about the seasonal fluctuation of other physical attributes in raccoons that may affect girth.

The BMI of feral raccoons in Kanagawa differed by season more obviously than the other two external measurements examined here; thus, fluctuations of BMI may be useful for assessing body fat deposition. Furthermore, the BMI of feral raccoons was found to correlate positively with seasonal changes of serum leptin, which is considered a marker of nutritional condition [36]. Because variations in body size in individuals affect BW, a standardized index based on body size was developed for assessing body fat deposition in mammals $[5,15,18,27,30,33]$. Nevertheless, Pitt et al. [31] reported that the best index, which is characterized by a modified ratio of body mass to length (body mass $/ \mathrm{BL}^{2}$ ), explained only $62 \%$ of the observed variation in fat determined using BIA for raccoons; thus, morphological condition indices alone were concluded to be unsatisfactory for estimating body condition. Here, we therefore also visually assessed relative body fat deposition in adult feral raccoons as VFI and examined the relationship between VFI and external measurements.

The means of BW, GM, and BMI differed significantly between VFI grades, a finding that is consistent with the hypothesis that these measurements change depending on the body fat deposits of raccoons. The results of discriminant analysis indicate that VFI I raccoons can be distin- guished from higher VFI grades, as a highly discriminant function ratio was obtained; however, discriminant analysis for distinguishing between VFI II and VFI III is not reliable. These results suggest that only the low fat deposit group would be distinguishable from medium or high groups of feral raccoons in Kanagawa Prefecture by the method of discriminant analysis based on BW, GM, and BMI. Thus, we conclude that discriminant analysis using these external measurements should not be applied to distinguish between medium and high fat deposit groups. However, BMI was the most valuable component for assessing body fat deposition by structure matrix values. Based on the observed seasonal changes of our data and the results of a previous study [36], we consider that the BMI of adult feral raccoons is a reliable indicator for body fat deposition.

If researchers are interested in determining the nutritional status of individual animals, it is necessary to evaluate body fat deposition by BIA or direct methods. Ideally, a costeffective method should be selected for assessing nutritional status in large sample sizes and over the long term. For understanding the population dynamics of wildlife, longterm sampling using the identical physiological indices throughout would enhance the reliability of conclusions drawn from the collected data [14]. Due to the many advantages of BIA [31], this method may be introduced in Japan to assess the body fat deposition of feral raccoons in the future. However, our results suggest that researchers and wildlife managers should use a relative index for assessing body fat deposition based on BMI in the absence of the BIA method because the fat deposition of feral raccoon populations is considered to be an important factor for scientific and practical management of this invasive species.

In conclusion, our findings suggest that BMI may serve as a complementary index for assessing relative body fat deposition of adult feral raccoons in Kanagawa Prefecture, and may be generalizable to populations in other areas. In North America, Fritzell et al. [11] demonstrated that the low pregnancy rate of raccoons was related to low physical condition using the $\mathrm{BW}$ as the condition indicator. On the other hand, it has been considered that low physical condition of raccoons might increase an individual's probability of contracting a disease upon exposure [33]. Contrary to these reports, if the population in Japan is concerned about high reproductive rate and low mortality with relation to the high fat deposit group of raccoons, we should control the factors including food sources that cause high fat deposition or conduct a more extensive eradication of wild raccoon populations. For example, Prange et al. [33] suggested that the effective methods for controlling of fat deposit of raccoons were the reduction or elimination of access to anthropogenic food sources such as refuse. Our results indicated that assessing fat deposition using BMI was more appropriate than that using BW. Therefore, future management programs should examine the BMI, as it potentially serves as an indicator for reproductive status and/or mortality in invasive raccoon populations. 
ACKNOWLEDGMENTS. The authors would like to sincerely thank Dr. T. Tsutsui (International Institute of Small Animal Medicine (BioPlus), AHB international Inc., Tokyo WEC); Dr. E. Kawakami, and Dr. H. Suzuki (Department of Veterinary, Nippon Veterinary and Life Science University); and Dr. M. Asano (Faculty of Applied Biological Sciences, Gifu University) for their helpful suggestions. We wish to thank the Kanagawa government office for its cooperation in building a raccoon retrieval system, and the Kamakura, Yokohama, Yokosuka, and Hayama local government offices for providing captured raccoons. We would also like to express our sincere gratitude to Mr. M. Kaneda; Mr. S. Kato, President of Strain Co., Ltd., and Mr. S. Kumagai, President of Kawasaki Shin-ei Kankyo Co., Ltd. for helping us retrieve raccoons and providing important information about the captured raccoons. We are also grateful to the members of Laboratory of Wildlife Medicine, Nippon Veterinary and Life Science University for their assistance with analyses. This study was financially supported by a grant from the Research Project for Utilizing Advanced Technologies in Agriculture, Forestry, and Fisheries (Project No. 18001). We thank ThinkSCIENCE K.K., Tokyo for editorial assistance.

\section{REFERENCES}

1. Asano, M., Matoba, Y., Ikeda, T., Suzuki, M., Asakawa, M. and Ohtaishi, N. 2003. Growth pattern and seasonal weight changes of the feral raccoon (Procyon lotor) in Hokkaido, Japan. Jpn. J. Vet. Res. 50: 165-173.

2. Asano, M., Matoba, Y., Ikeda, T., Suzuki, M., Asakawa, M. and Ohtaishi, N. 2003. Reproductive characteristics of the feral raccoon (Procyon lotor) in Hokkaido, Japan. J. Vet. Med. Sci. 65: 369-373.

3. Atkinson, S. N. and Ramsay, M. A. 1995. The effects of prolonged fasting of the body composition and reproductive success of female polar bears (Ursus maritimus). Funct. Ecol. 1995: 559-567.

4. Cattet, M. 1990. Predicting nutritional condition in black bears and polar bears on the basis of morphological and physiological measurements. Can. J. Zool. 68: 32-39.

5. Cattet, M. R. L., Caulkett, N. A., Obbard, M. E. and Stenhouse, G. B. 2002. A body-condition index for ursids. Can. J. Zool. 80: $1156-1161$.

6. Cavallini, P. 1996. Comparison of body condition indices in the red fox (Fissipedia, Canidae). Mammalia 60: 449-462.

7. Elowe, K. D. and Dodge, W. E. 1989. Factors affecting black bear reproductive success and cub survival. J. Wildl. Manage. 53: 962-968.

8. Evsikov, V. I., Nazarova, G. G. and Muzyka, V. Y. 2008. Body condition and reproductive characteristics of female water voles (Arvicola terrestris L.). Russian J. Ecol. 39: 414-417.

9. Farley, S. D. and Robbins, C. T. 1994. Development of two methods to estimate body composition of bears. Can. J. Zool. 72: 220-226.

10. Fleharty, E. D., Krause, M. E. and Stinnett, D. P. 1973. Body composition, energy content, and lipid cycles of four species of rodents. J. Mammal. 54: 426-438.

11. Fritzell, E. K., Hubert, G. F. J., Meyen, B. E. and Sanderson, G. C. 1985. Age-specific reproduction in Illinois and Missouri raccoons. J. Wildl. Manage. 7: 60-73.

12. Gehrt, S. D. and Fritzell, E. K. 1999. Growth rates and intraspecific variation in body weights of raccoons (Procyon lotor) in southern Texas. Am. Midl. Nat. 141: 19-27.

13. Grau, C. A., Sanderson, G. C. and Rogers, J. P. 1970. Age determination of raccoons. J. Wildl. Manage. 34: 364-372.

14. Harder, J. D. and Kirkpatrick, R. L. 1996. Physiological methods in wildlife research. pp. 275-306. In: Research and Management Techniques for wildlife and habitats (Bookhout, T. A. ed.), The Wildlife Society, Maryland.

15. Hayama, S. and Matsubara, Y. 2010. Evaluation of indices for the assessment of fat deposition in Tsushima leopard cats (Prionaliurus bengalensis euptilurus). Jpn. J. Zoo Wildl. Med. 15: $57-64$.

16. Hellgren, E. C., Rogers, L. L. and Seal, U. S. 1993. Serum chemistry and hematology of black bears: physiological indices of habitat quality or seasonal patterns? J. Mammal. 74: 304-315.

17. Hilderbrand, G. V., Farley, S. D. and Robbins, C. T. 1998. Predicting body condition of bears via two field methods. J. Wildl. Manage. 62: 406-409.

18. Huot, J., Poulle, M. L. and Crete, M. 1995. Evaluation of several indices for assessment of coyote (Canis latrans) body composition. Can. J. Zool. 73: 1620-1624.

19. Ikeda, T., Asano, M., Matoba, Y. and Abe, G. 2004. Present status of invasive alien raccoon and its impact in Japan. Global Environ. Res. 8: 125-131.

20. Japan Veterinary Medical Association. 2007. Guidelines for the Management of Invasive Alien Species. The Wildlife Committee Report Division of Small Animal Medicine in JVMA (in Japanese).

21. Johnson, A. S. 1970. Biology of the Raccoon (Procyon lotor varius Nelson and Goldman), in Alabama (Bulletin 402). Auburn University Agricultural Experiment Station.

22. Junge, R. E. and Hoffmeister, D. F. 1980. Age determination in raccoons from cranial suture obliteration. J. Wildl. Manage. 44: 725-729.

23. Kanagawa Government. 2006. Kanagawa Raccoon Control Program (in Japanese).

24. Kato, T., Ichida, Y., Tei, K., Asano, M. and Hayama, S. 2009. Reproductive characteristics of feral raccoons (Procyon lotor) captured by the pest control in Kamakura, Japan. J. Vet. Med. Sci. 71: 1473-1478.

25. Kauhala, K. 1996. Reproductive strategies of the raccoon $\operatorname{dog}$ and the red fox in Finland. Acta Theriologica 41: 51-58.

26. Ladine, T. A. 1998. Relationship of mass to girth in raccoons, Procyon lotor (Mammalia: Procyonidae), from West Tennessee. Brimleyana 25: 91-98.

27. Lefebvre, C., Crête, M., Huot, J. and Patenaud, R. 1999. Prediction of body composition of live and post-mortem red foxes. J. Wildl. Dis. 35: 161-170.

28. Mech, L. D., Barnes, D. M. and Tester, J. R. 1968. Seasonal weight changes, mortality, and population structure of raccoons in Minnesota. J. Mammal. 49: 63-73.

29. Mugaas, J. N. and Seidensticker, J. 1993. Geographic variation of lean body mass and a model of its effect on the capacity of raccoon to fatten and fast. Bull. Fl. Mus. Nat. His. 36: 85-107.

30. Noyce, K. V., Coy, P. L. and Garshelis, D. L. 2002. Bone prominence and skin-fold thickness as predictors of body fat and reproduction in American black bears. Ursus 13: 275-284.

31. Pitt, J. A., Larivière, S. and Messier, F. 2006. Condition indices and bioelectrical impedance analysis to predict body condition of small carnivores. J. Mammal. 87: 717-722. 
32. Pitt, J. A., Larivière, S. and Messier, F. 2008. Survival and body condition of raccoon at the edge of the range. J. Wildl. Manage. 72: 389-395.

33. Prange, S., Gehrt, S. D. and Wiggers, E. P. 2003. Demographic factors contributing to high raccoon densities in urban landscapes. J. Wildl. Manage. 67: 324-333.

34. Prestrud, P. and Nilssen, K. 1992. Fat deposition and seasonal variation in body composition of arctic foxes in Svalbard. $J$. Wildl. Manage. 56: 221-233.

35. Sanderson, G. C. and Nalbandov, A. V. 1973. The reproductive cycle of the raccoon in Illinois. Ill. Nat. Hist. Surv. Bull. 31: $29-85$.

36. Shibata, H., Akahane, R., Honjoh, T., Asano, M., Mominoki, K., Fujii, K., Suzuki, M., Ohtaishi, N., Ishioka, K., Ahmed, M., Soliman, M., Kimura, K. and Saito, M. 2005. Seasonal changes in serum leptin of the feral raccoon (Procyon lotor) determined by canine-leptin-specific ELISA. J. Exp. Zool. A Comp. Exp. Biol. 303: 527-533.

37. Stringer, E. M., Stoskopf, M. K., Simons, T., O'Connell, A. F. and Waldstein, A. 2010. Ultrasonic measurement of body fat as a means of assessing body condition in free-ranging raccoons (Procyon lotor). Int. J. Zool 2010: Article ID 972380, 972386 pages.

38. Stuewer, F. W. 1943. Raccoons: their habitat and management in Michigan. Ecol. Monogr. 13: 203-257.

39. Vaughan, M. R. 2002. Oak trees, acorns, and bears. pp. 224 240. In: Oak Forest Ecosystems: Ecology and Management for Wildlife (McShea, W. J. and W. M. Healy, eds.), Johns Hopkins University Press, Baltimore.

40. Virgl, J. A. and Messier, F. 1992. Seasonal variation in body composition and morphology of adult muskrats in central Saskatchewan. Can. J. Zool. 228: 461-477. 